\title{
pwrEWAS: a user-friendly tool for comprehensive power estimation for epigenome wide association studies (EWAS)
}

\author{
Stefan Graw ${ }^{1 *}$, Rosalyn Henn ${ }^{2}$, Jeffrey A. Thompson ${ }^{1}$ and Devin C. Koestler ${ }^{1}$
}

\begin{abstract}
Background: When designing an epigenome-wide association study (EWAS) to investigate the relationship between DNA methylation (DNAm) and some exposure(s) or phenotype(s), it is critically important to assess the sample size needed to detect a hypothesized difference with adequate statistical power. However, the complex and nuanced nature of DNAm data makes direct assessment of statistical power challenging. To circumvent these challenges and to address the outstanding need for a user-friendly interface for EWAS power evaluation, we have developed pwrEWAS.
\end{abstract}

Results: The current implementation of pwrEWAS accommodates power estimation for two-group comparisons of DNAm (e.g. case vs control, exposed vs non-exposed, etc.), where methylation assessment is carried out using the Illumina Human Methylation BeadChip technology. Power is calculated using a semi-parametric simulation-based approach in which DNAm data is randomly generated from beta-distributions using CpG-specific means and variances estimated from one of several different existing DNAm data sets, chosen to cover the most common tissue-types used in EWAS. In addition to specifying the tissue type to be used for DNAm profiling, users are required to specify the sample size, number of differentially methylated CpGs, effect size(s) $\left(\Delta_{\beta}\right)$, target false discovery rate (FDR) and the number of simulated data sets, and have the option of selecting from several different statistical methods to perform differential methylation analyses. pwrEWAS reports the marginal power, marginal type I error rate, marginal FDR, and false discovery cost (FDC). Here, we demonstrate how pwrEWAS can be applied in practice using a hypothetical EWAS. In addition, we report its computational efficiency across a variety of user settings.

Conclusion: Both under- and overpowered studies unnecessarily deplete resources and even risk failure of a study. With pwrEWAS, we provide a user-friendly tool to help researchers circumvent these risks and to assist in the design and planning of EWAS.

Availability: The web interface is written in the R statistical programming language using Shiny (RStudio Inc., 2016) and is available at https://biostats-shinyr.kumc.edu/pwrEWAS/. The R package for pwrEWAS is publicly available at GitHub (https://github.com/stefangraw/pwrEWAS).

Keywords: DNA methylation, Microarray data analysis, Statistical power, Sample size calculation, Bioconductor package, Illumina human methylation BeadChip

\footnotetext{
* Correspondence: sgraw@kumc.edu

${ }^{1}$ Department of Biostatistics \& Data Science, University of Kansas Medical

Center, Kansas City, KS, USA

Full list of author information is available at the end of the article
}

(c) The Author(s). 2019 Open Access This article is distributed under the terms of the Creative Commons Attribution 4.0 International License (http://creativecommons.org/licenses/by/4.0/), which permits unrestricted use, distribution, and reproduction in any medium, provided you give appropriate credit to the original author(s) and the source, provide a link to the Creative Commons license, and indicate if changes were made. The Creative Commons Public Domain Dedication waiver (http://creativecommons.org/publicdomain/zero/1.0/) applies to the data made available in this article, unless otherwise stated. 


\section{Background}

Epigenome-wide association studies (EWAS) aim to examine the relationship between epigenetic marks and exposure(s) or phenotype(s) on a genome-wide level. DNA methylation (DNAm) is the most widely studied epigenetic mechanism and involves the chemical addition of a methyl group to the 5-carbon position of cytosine in the context of cytosine-phosphate-guanine (CpG) dinucleotides. The vast majority of EWAS use microarray-based platforms for assessing DNAm, such as the Illumina Infinium HumanMethylation BeadArrays (Illumina Inc.), as these platforms provide a compromise between coverage, cost, and sample throughput $[1,2]$. Illumina's latest methylation microarrays, the Infinium HumanMethylation450 and Infinium HumanMethylationEPIC, interrogate the methylation levels of over 450,000 and 850,000 CpG dinucleotides, respectively. While these arrays differ in their coverage, both allow for the assessment of methylation at single-nucleotide resolution, quantified using what is referred to as the methylation $\beta$-value, an approximately continuously-distributed measure that reflects the methylation extent of a specific CpG locus; ranging from 0 (unmethylated) to 1 (methylated). Interest in studying DNAm in the context of human health and disease has been ignited by the now numerous studies that have reported altered patterns of DNAm across various human diseases $[3,4]$ and in response to environmental exposures [5], along with reversible nature of DNAm, which makes it a promising target for potential treatments and therapies [6]. To detect a hypothesized difference in DNAm with adequate statistical power it is crucial to assess the required sample size. However, the complex nature of DNAm data $[7,8]$ makes a direct power assessment challenging, as power depends on several factors: planned study sample size, array technology used to profile DNAm, tissue type used in assessing DNAm, proportion of differentially methylated $\mathrm{CpGs}$ and the distribution of their differences $\left(\Delta_{\beta}\right)$, and multiplicity.

The importance of formal power assessment and sample size justification in the design of research studies has been recognized and addressed in related omics fields, and motivated the development of power evaluation tools, including: "RNAseqPS" [9], "RNASeqPowerCalculator" [10] and "PROPER" [11] for RNA-Seq data, and "CaTs" [12], "Statistical Power Analysis tool" [13], "GWAPower" [14], and "SurvivalGWAS_Power" [15] for GWAS data. However, surprisingly little attention has been given to this topic in the context of EWAS and while there has been substantial work on the development of statistical methods and publicly available software for the preprocessing, quality control, normalization, and analysis of DNA methylation data $[16,17]$, methods and tools for power evaluation for EWAS are lagging. Consequently, most EWAS are conducted in the absence of formal power analyses, resulting in studies that are potentially under- or overpowered [18]. To our knowledge, only three studies have formally addressed the issue of power evaluation in the context of EWAS [19-21]. Wang et al. [21] simulated DNAm data for two group comparisons from uniform-normal mixture distributions with parameter settings that capture three general types of distributions often seen in methylation data (methylated, unmethylated, and partially methylated). Power was then assessed and compared for two differential methylation detection methods: proposed method by Wang et al. [21] and t-tests. Rakyan et al. [20] generated DNAm data for two group comparisons from single and mixture beta distributions in three scenarios with four effect sizes each and differences in methylation ranging from 1.25 to $14.4 \%$. Logistic regression was then applied to assess differential methylation and power was evaluated. Finally, Tsai et al. [19] simulated DNAm data for two group comparisons from nine single locus DNAm distributions, again falling into three categories: methylated, hemi-methylated and unmethylated. The expected differences in methylation ranged from 1 to $60 \%$. Differential methylation was then analyzed by t-tests and Wilcoxon rank-sum tests, and the respective power was assessed.

All three approaches utilize a limited number of single locus distributions, which result in a wide range of methylation levels of CpG sites, but may lead to unrealistic data with a predefined fixed number of expected differences in methylation between two groups. This is because individual CpGs have their own unique mean and variance depending on their genomic context and susceptibility to become methylated and vary depending on the tissue type used for methylation assessment [22]. Analogously, expected differences in CpG-specific methylation between two or more groups are expected to come from a continuous distribution instead of having predefined discrete values [23]. In addition to the potential limitations above, none of the previously described methods provided accompanying software for their methodology, limiting their application within the epigenomics-research community. Therefore, there remains an outstanding need for publicly available software that addresses these limitations and enables comprehensive assessments of statistical power in the context of EWAS involving CpG-specific comparisons of DNAm.

Inspired by PROPER [11], a publicly available tool to assist researchers with power assessment in RNA-seq studies, we have developed pwrEWAS for comprehensive power evaluation in the context of case-control EWAS. In pwrEWAS, power is estimated using semi-parametric simulation-based approach. First, DNAm data is randomly generated for each comparator 
group based on user-supplied information concerning the expected fraction of differentially methylated CpGs between groups and their expected effect size $\left(\Delta_{\beta}\right)$. To simulate realistic methylation data, DNAm data are generated from a beta-distribution using CpG-specific means and variances estimated from one of several different publicly available DNAm data sets, chosen to span the most common tissue-types used in EWAS. This gives the user the flexibility to select the tissue type (e.g., whole blood, peripheral blood mononuclear cells (PBMCs), etc.) that is most appropriate for the study being planned. Next, the generated data undergoes a formal differential methylation analysis, the results of which are used to estimate statistical power. In what follows, we begin by describing the statistical framework underlying pwrEWAS, followed by its demonstration and an assessment of its run time across different user settings. We finish with a discussion of the limitations of pwrEWAS and describe future extensions.

\section{Methods}

As previously mentioned, the Illumina Infinium HumanMethylationEPIC microarray measures the methylation status of $>850,000 \mathrm{CpGs}$ throughout the genome. For a single $\mathrm{CpG}, \mathrm{DNAm}$ is quantified via the $\beta$-value, $\beta$ $=\frac{M}{M+U}$, where $M$ and $U$ are the methylated and unmethylated signal intensities, respectively. As $M$ and $U$ are typically assumed to be gamma-distributed random variables with equal scale parameter [7], it follows that the $\beta$-value follows a beta-distribution. As such, the $\beta$-value ranges from 0 to 1 and represents the methylation extent for a specific CpG. Under ideal conditions, a $\beta$-value of zero signifies that all alleles in all cells of a sample were unmethylated at that $\mathrm{CpG}$ site, while a $\beta$-value of one indicates methylation throughout all alleles in all cells at that CpG site [24]. A common goal of EWAS is to identify CpG-specific differential methylation based on some phenotype or exposure. Formally, this involves testing the null hypothesis $H_{0}: \Delta_{\beta, j}=0$, where $\Delta_{\beta, j}=\mu_{j}^{(1)}-\mu_{j}^{(2)}$ and represents the difference in mean methylation at the $j^{\text {th }}$ CpG between two groups (e.g. cases versus controls, exposed versus unexposed, etc.), with $j=\{1, \ldots, J\}$ and $J$ representing the number of interrogated CpGs.

pwrEWAS is written using the $\mathrm{R}$ statistical programming language (http://r-project.org) and is comprised of three major steps: (1) data generation, (2) differential methylation analysis, and (3) power evaluation (Fig. 1). Users are required to provide input parameters, including: tissue type to be used for methylation assessment, assumed total sample size (can be specified as a range of possible sample sizes), percentage of the total sample split into two groups (50\% corresponds to a balanced study), number of CpGs to be formally tested, expected number of differentially methylated CpGs, and the expected difference in methylation between the comparator groups $\left(\Delta_{\beta}\right)$ or alternatively, the standard deviation of these differences $\left(s d\left(\Delta_{\beta}\right)\right)$.

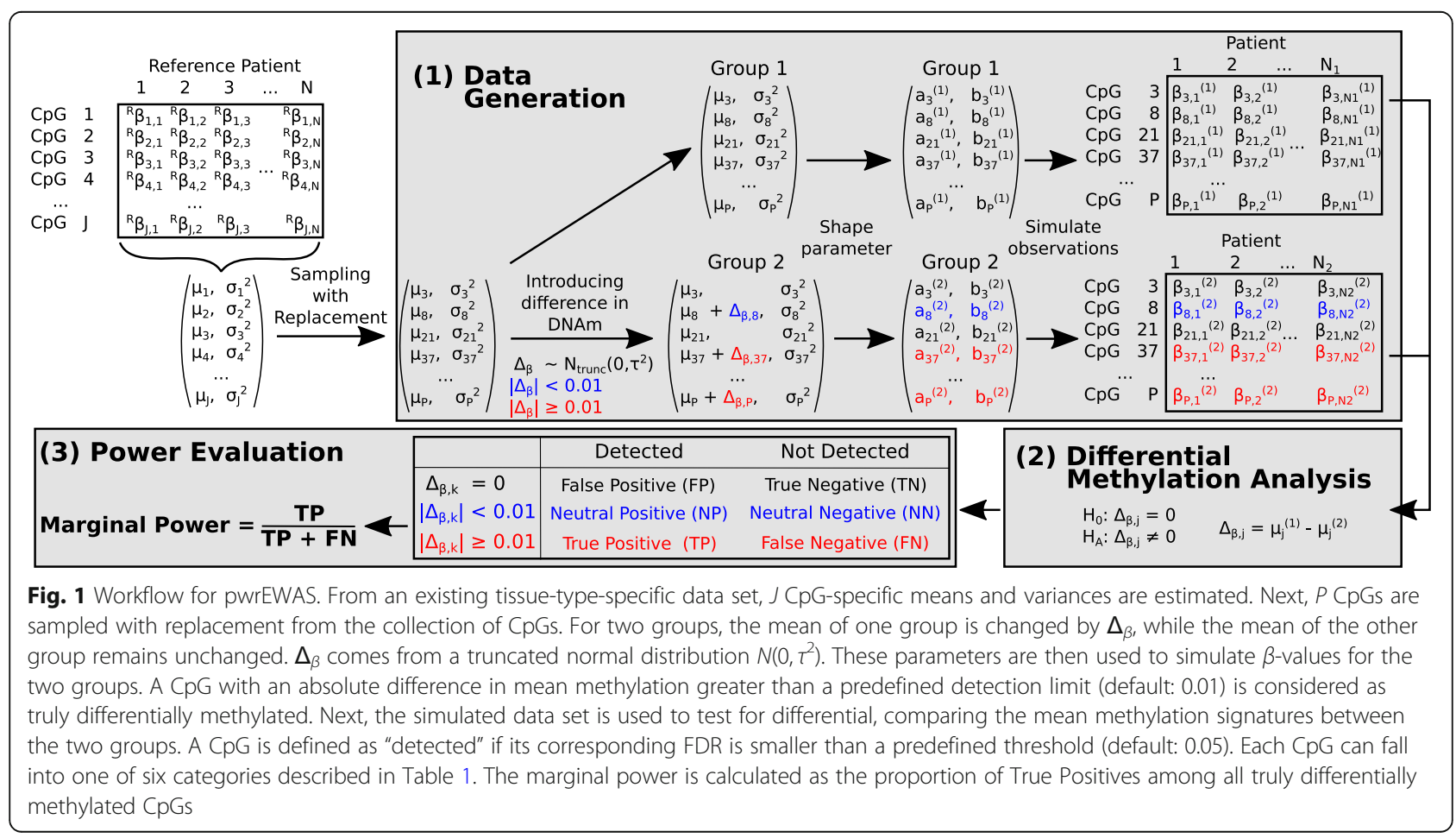


To assist users with their experimental design, pwrEWAS provides estimates of statistical power as a function of the assumed sample and effect size(s). Further, it provides estimates of the marginal type I error rate, marginal FDR, false discovery cost (FDC), the distribution of simulated $\Delta_{\beta}$ 's, and probabilities of identifying at least one true positive. The probability of identifying at least one true positive is beneficial in studies where either the effect or sample size is very small (e.g. pilot or explanatory studies).

\section{Data generation}

Our approach to estimating statistical power begins by leveraging publicly available DNA methylation data sets in order to simulate realistic methylation data. Data sets used for the purpose of simulation were selected to represent the most commonly used tissue types used in EWAS. To identify these tissue types, the Gene Expression Omnibus (GEO) data repository was manually scanned and tissue types were rank-ordered based on the number of GEO deposited data sets including Illumina Infinium Human Methylation BeadChip data for that tissue type. For each of the most common tissue types identified, a single representative data set was selected (Table 1). Representative datasets were selected based on a combination of the study's sample size (preference toward larger data sets), study design, and the inclusion of DNA methylation profiles for healthy, non-diseased subjects.

For each selected tissue type, CpG-specific means and variances were estimated $\left(\hat{\mu}_{j}=\frac{1}{N} \sum_{i=1}^{N} \beta_{i, j}\right.$ and $\hat{\sigma}_{j}^{2}=\frac{1}{N-1}$ $\left.\sum_{i=1}^{N}\left(\beta_{i, j}-\hat{\mu}_{j}\right)^{2}\right)$, where $\beta_{i, j}$ represents the methylation $\beta$-value for CpG $j=\{1, \ldots$,$\} in subject i=\{1, \ldots, N\}$. CpG-specific parameter estimates are then used as the basis for simulating realistic methylation data using a semi-parametric simulation strategy. First, $P$ pairs of CpG-specific means and variances $\left(\hat{\mu}_{j}, \hat{\sigma}_{j}^{2}\right)$ are sampled with replacement from one of the tissue-type specific reference data sets (Table 1 ). By default, $P$ is set to 100,000 CpG sites, as previous studies have suggested filtering out low-variable CpGs to offset the burden of multiplicity [25], however in principle, $P$ can be set according to the user's preference (e.g., $P=866,836$ for EWAS conducted using the EPIC array). Thus, pwrEWAS allows up- or down-scaling to any number of CpGs that the investigator plans to measure and conducted differential methylation analyses on. This is an important feature since the EPIC array is the successor to the now discontinued Infinium HumanMethylation450 array, which represents the technology used for methylation assessment of the tissue-specific reference data sets used as the basis of our simulation strategy. Of the $P$ sampled CpGs, a difference in mean DNAm $\left(\Delta_{\beta}\right)$ is imposed on $K$ CpGs, where $K \leq P$. The number of differentially methylated CpGs, $K$, is selected by the user and ideally motivated by a pilot study, previous literature, or expert knowledge about the effect of the phenotype(s) or exposure(s) of interest on DNA methylation. The mean methylation of $K$ CpGs is shifted in one of the comparator groups by $\Delta_{\beta}=\left\{\Delta_{\beta}, 1, \ldots \Delta_{\beta}, k, \ldots \Delta_{\beta}, K\right\}$, while the mean methylation in the other comparator group remains unchanged. Due to the nature of $\beta$-values and the parameter restrictions of the beta distribution $\left(0 \leq \mu_{k} \leq 1\right.$ and $\left.0<\sigma_{k}^{2}<0.25\right), \Delta_{\beta, k}$ is bounded by $\frac{1}{2}-\mu_{k}$ $\pm \sqrt{\frac{1}{4}-\sigma_{k}^{2}}$, where $\mu_{k}$ and $\sigma_{k}^{2}$ are CpG-specific means and variances, respectively (see Additional file 1 for additional details). Due to its boundedness, $\Delta_{\beta, k}$ is drawn from a truncated normal distribution $\left(\Delta_{\beta}, k \sim N_{k}\left(0, \tau^{2}\right)\right)$. The normal distribution was chosen based on observed differences in DNAm of differentially methylated CpGs in previously published EWAS (see Additional file 2: Figure S1). The standard deviation of the simulated differences $\tau$ can be provided by the user or be automatically be

Table 1 Curated tissue-type specific DNAm data sets used by pwrEWAS

\begin{tabular}{|c|c|c|c|}
\hline Tissue Type & Accession Number & Subjects within GSE-ID limited to & Reference \\
\hline Saliva & GSE92767 & & [38] \\
\hline Lymphoma & GSE42372 & disease state: non-HIV lymphoma & [39] \\
\hline Placenta & GSE62733 & health state: Normal & [40] \\
\hline Liver & GSE61258 & diseasestatus: Control & [41] \\
\hline Colon & GSE77718 & disease state: Normal & [42] \\
\hline Blood (Adults) & GSE42861 & subject: Normal & {$[43,44]$} \\
\hline Blood (Children) & GSE83334 & age: 5 years & [45] \\
\hline Blood (Newborns) & GSE82273 & & [46] \\
\hline Cord-blood (whole blood) & GSE69176 & & \\
\hline Cord-blood (PBMC) & GSE110128 & cord blood & [47] \\
\hline Adult (PBMC) & GSE67170 & disease state: control & [48] \\
\hline
\end{tabular}

Representative data sets for the most commonly used tissue types for EWAS with inclusion criteria for subjects 
determined based on the user-specified target $\Delta_{\beta}$ and the expected number of differentially methylated CpGs, such that $\Delta_{\beta}$ matches the target maximal difference in mean methylation. To achieve this, an internal function simulates $P \Delta_{\beta, k}$ 's (this matches the number of subsequently simulated CpGs) 100 times, while stepwise adjusting $\tau$. The goal is to identify a standard deviation $\tau$ for the truncated normal distribution to matches the targeted maximal difference in DNAm. Therefore, $\tau$ is adjusted stepwise until the 99.99th percentile of the absolute value of simulated $\Delta_{\beta, k}$ 's falls within a range around the targeted maximal difference in DNAm. The range is equal to the detection limit $( \pm 0.005$ based on default detection limit: 0.01). (Additional file 2: Figure S2) shows the distribution of simulated $\Delta_{\beta, k}$ 's for different effect sizes and its respective range that the 99.99th percentile of the simulated $\Delta_{\beta, k}$ 's needs to fall in for $\tau$ to be accepted.

Since $\Delta_{\beta}$ is simulated from a truncated normal distribution, a certain proportion of $\Delta_{\beta}$ are within the detection limit range around zero and thus, do not exhibit a biologically meaningful difference in mean methylation. To ensure that $K$ includes the number of meaningfully differential methylated CpGs (truly differentially methylated $\mathrm{CpGs),} K$ is calculated to reflect the user-supplied target number of differentially methylated CpGs ( $K$ $=\frac{1}{\text { Percentage of truly DM CpGs }} *$ Target number of DMCpGs ). This results in $K$ CpGs with changed means $\left(\Delta_{\beta, k} \neq 0\right)$ and $P-K$ CpGs with unchanged means $\left(\Delta_{\beta, k}=0\right)$ between the two comparator groups. Variances across all $P$ CpGs remain unchanged in both comparator groups, that is, comparator groups are assumed to have the same CpG-specific variances. Next, the means and variances of both comparator groups are used to calculate CpG-specific shape parameters for the beta-distribution: $a_{j}=\mu_{j}^{2}\left(\frac{1-\mu_{j}}{\sigma_{j}^{2}}-\frac{1}{\mu_{j}}\right)$ and $b_{j}=a_{j}\left(\frac{1}{\mu_{j}}-1\right)$ (see Additional file $1)$. The two comparator group specific matrices $(P \times 2)$ containing the CpG-specific shape parameters are then used to generate $N_{1}$ and $N_{2}$ beta-distributed observations for each $\mathrm{CpG}$, for both comparator groups respectively, resulting in two matrices $\left(P \times N_{1}\right.$ and $\left.P \times N_{2}\right)$ of $\beta$-values, which are subsequently used for the differential methylation analysis.

Simulated CpGs fall into one of three categories: (1) not differentially methylated $\left(\Delta_{\beta, k}=0\right)$, (2) differentially methylated with negligible difference $\left(\left|\Delta_{\beta, k}\right|<0.01\right)$, and (3) truly differentially methylated $\left(\left|\Delta_{\beta, k}\right| \geq 0.01\right)$. The threshold of 0.01 was chosen according to the detection limit of DNAm arrays [8], but can be modified by the user.

\section{Differential methylation detection}

Following data generation, differential methylation analyses are carried out using one of several established parametric and nonparametric approaches, including: limma [26], CpGassoc [27], t-test, or a Wilcoxon rank-sum test. In the first three of the above methods, simulated $\beta$-values are first transformed to methylation $M$-values using the logit-transformation $\left(M=\log _{2}\left(\frac{\beta}{1-\beta}\right)\right)$ due to their assumption of normality [24, 28]. Each method reports CpG-specific $p$-values, which are multiplicity adjusted using the Benjamini and Hochberg method [29] to control the False Discovery Rate (FDR).

\section{Power assessment}

Tested CpGs fall into one of six categories: (1) TP (True Positive): detected CpGs with meaningful difference in mean DNAm, (2) NP (Neutral Positive): detected CpGs with negligible difference in mean DNAm, (3) FP (False Positive): detected $\mathrm{CpG}$ with no difference in mean DNAm, (4) TN (True Negative): undetected CpGs with no difference in mean DNAm, (5) NN (Neutral Negative): undetected $\mathrm{CpGs}$ with negligible difference in mean DNAm, and (6) FN (False Negative): undetected CpGs with meaningful difference in mean DNAm (Table 2).

Since it can be argued that CpGs with a negligible $\Delta_{\beta}$, ${ }_{k}$ are not biologically meaningful, we calculate the empirical marginal power, defined by $\mathrm{Wu}$ et al. [11] as the proportion of truly differentially methylated CpGs detected at the specified FDR threshold, $\frac{T P}{T P+F N}$ (Table 2). Further, even though failing to discover differentially methylated CpGs represents a type II error, failing to detect CpGs with a negligible $\Delta_{\beta, k}$ can be disregarded (NN) due to their likely unimportance. Additionally, as identifying CpGs with a negligible $\Delta_{\beta, k}(\mathrm{NP})$ is not as crucial as identifying CpGs with a biologically meaningful $\Delta_{\beta, k}$ (TP), we also report the false discovery cost ( $\left.F D C=\frac{F P}{T P}\right)[11]$.

For each of the assumed sample and effect sizes we report the following metrics, averaged across simulations to obtain reliable estimates:

- Empirical classical power: The ratio of correctly detected CpGs and all differentially methylated CpGs

$$
\text { classicalPower }=\frac{N P+T P}{N P+N N+T P+F N}
$$

- Empirical marginal power: The ratio of correctly detected CpGs with biologically meaningful differences and all differentially methylated CpGs with biologically meaningful differences (excluding 
Table 2 Differential methylation detection and terminology

\begin{tabular}{lllll}
\hline & Differentially Methylated & Truly Differentially Methylated & Detected & Not Detected \\
\hline$\Delta_{k}=0$ & No & No & False Positive (FP) & True Negative (TN) \\
$\left|\Delta_{k}\right|<0.01$ & Yes & No & Neutral Positive (NP) & Neutral Negative (NN) \\
$\left|\Delta_{k}\right| \geq 0.01$ & Yes & Yes & True Positive (TP) & False Negative (FN)
\end{tabular}

Each CpG can fall into one of six following categories: False Positive (FP; detected CpG with no simulated difference in mean methylation); Neutral Positive (NP; detected CpG with negotiable simulated difference in mean methylation); True Positive (TP; detected CpG with meaningful simulated difference in mean methylation); True Negative (TN; not detected CpG with no simulated difference in mean methylation); Neutral Negative (NN; not detected CpG with negotiable simulated difference in mean methylation); False Negative (FN; not detected CpG with meaningful simulated difference in mean methylation)

Neutral Positives and Neutral Negative with negligible differences):

marPower $=\frac{T P}{T P+F N}$

- Empirical marginal Type I Error: The ratio of wrongly detected CpGs and all CpGs with no difference

marTypeI $=\frac{F P}{F P+T N}$

- Empirical False Discovery Rate (FDR): The ratio of wrongly detected CpGs and all detected CpGs

$F D R=\frac{F P}{F P+N P+T P}$

- Empirical False Discovery cost (FDC): The ratio of wrongly detected CpGs and correctly detected CpGs:

$$
F D C=\frac{F P}{T P}
$$

\section{Visualization}

The pwrEWAS package contains two functions that can be used to visualize the results ("pwrEWAS_powerPlot" and "pwrEWAS_deltaDensity"). "pwrEWAS_powerPlot" displays the estimated power as a function of sample size with error bars (2.5th and 97.5th percentile calculated across simulations). Power across different target $\Delta_{\beta}^{\prime}$ s as a function of sample size is differentiated by different colors (Fig. 2, Box 4). "pwrEWAS_deltaDensity" illustrates the distribution of simulated $\Delta_{\beta, \mathrm{k}}$ 's for different target $\Delta_{\beta}^{\prime}$ s as density plots (Fig. 2, Box 7). Densities for different target $\Delta_{\beta}^{\prime} \quad \mathrm{s}$ are color-coded as well and match the colors of the power curve ("pwrEWAS_powerPlot").

\section{Results}

Consider a hypothetical study that aims to understand the relationship between electronic cigarettes (e-cigarette) and DNAm derived from adult blood. The use of e-cigarettes has increased dramatically over the last decade, especially among young adults [30]. There exists a common perception in the population, including pregnant women and women in child-bearing age, that e-cigarettes are less harmful than smoking tobacco cigarettes [31]. Although, studies have reported the presence of toxic components in e-cigarette aerosol [30], there presently exists no study investigating the relationship between e-cigarette and DNAm derived from adult human blood. As the effect of e-cigarette usage on DNAm is presently unknown, but is of interest in this hypothetical study, we will use the previously reported effects of tobacco smoke on blood-derived DNAm as an upper limit for the effect of e-cigarette usage on DNAm. Previous studies analyzing the effect of smoking tobacco cigarettes on blood-derived patterns of DNA methylation have reported CpG-specific differences up to $24 \%$ between smokers and non-smokers, with a wide range of CpGs $(724-18,760)$ declared as significantly differentially methylated (FDR $\leq 0.05$ ) [32-34]. Hence, we want to investigate the number of subjects required to detect DNAm differences in 2500 CpGs (selected to be within the range of the number of significantly differently methylated CpGs reported between smokers and non-smokers in previous reports) with $80 \%$ power for three reasonable effect sizes $\left(\Delta_{\beta}=\{0.10,0.15,0.20\}\right.$ and one deliberately small effect size $\Delta_{\beta}=0.02$, representing differences in DNAm up to $\sim 2 \%, \sim 10 \%, \sim 15 \%$ and $\sim 20 \%$ ). To cover a wide range of total sample sizes, we analyzed total sample sizes ranging from 20 to 260 individuals with increments of 40 and equal allocation between e-cigarette users and non-users, while keeping the remaining default parameters of pwrEWAS intact: 


\section{pwrEWAS}

pwrEWAS is a computationally efficient tool to estimate power in EWAS as a function of sample and effect size for two-group comparisons of DNAm (e.g., case vs control, exposed vs non-exposed, etc.). Detailed description of in-/outputs, instructions and an example, as well as interpretations of the example results are provided in the following vignette: pwrEWAS vignette

\section{3}

Authors: Stefan Graw, Devin Koestle Department of Biostatistics, University of Kansas School of Medicine

\begin{tabular}{l} 
Tissue Type \\
\hline Blood adult \\
Minimum total sample size \\
\hline 20 \\
Maximum total sample size \\
\hline 260 \\
\hline Sample size increments \\
\hline 40 \\
\hline Samples rate for group 1 \\
\hline 0.5 \\
\hline Number of CpGs tested \\
\hline 100000 \\
\hline Target number of DM CPGs \\
\hline 2500 \\
\hline 0.05 \\
\hline Target max $\Delta$ \\
\hline (comma delimited) \\
\hline $0.02,0.10,0.15,0.20$ \\
\hline SD $(\Delta)$ \\
\hline
\end{tabular}

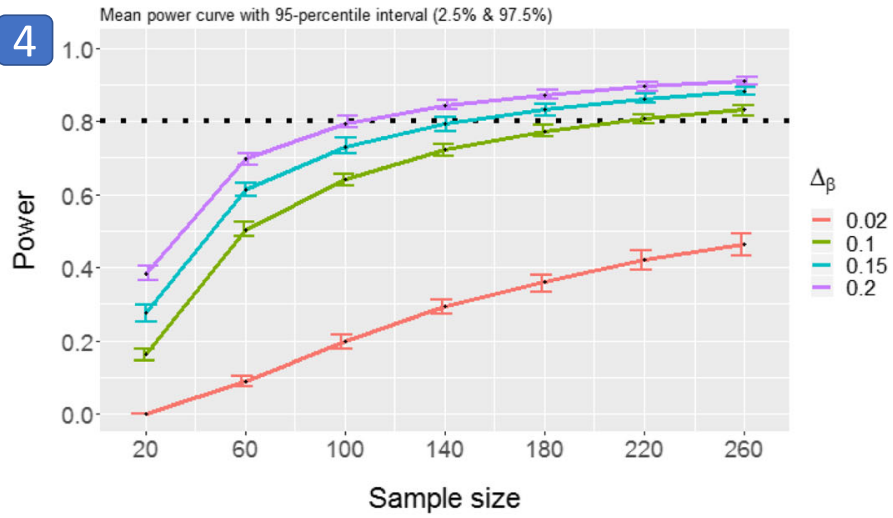

च Advanced settings

\begin{tabular}{lllll}
\hline 5 & & & & \\
\cline { 5 - 5 } $\mathrm{N} \backslash \Delta_{\beta}$ & $\mathbf{0 . 0 2}$ & $\mathbf{0 . 1}$ & $\mathbf{0 . 1 5}$ & 0.2 \\
\hline 20 & 0 & 0.16 & 0.28 & 0.38 \\
\hline 60 & 0.09 & 0.5 & 0.61 & 0.7 \\
\hline 100 & 0.2 & 0.64 & 0.73 & 0.79 \\
\hline 140 & 0.29 & 0.72 & 0.79 & 0.84 \\
\hline 180 & 0.36 & 0.77 & 0.83 & 0.87 \\
\hline 220 & 0.42 & 0.81 & 0.86 & 0.9 \\
\hline 260 & 0.46 & 0.83 & 0.88 & 0.91 \\
\hline
\end{tabular}

\begin{tabular}{lllll}
$\mathbf{6} 6$ & & & & \\
$\mathrm{~N} \backslash \Delta_{\boldsymbol{\beta}}$ & $\mathbf{0 . 0 2}$ & $\mathbf{P}(\# \mathrm{TP} \geq 1)$ & & \\
\hline 20 & 0.36 & 1 & $\mathbf{0 . 1 5}$ & $\mathbf{0 . 2}$ \\
\hline 60 & 1 & 1 & 1 & 1 \\
\hline 100 & 1 & 1 & 1 & 1 \\
\hline 140 & 1 & 1 & 1 & 1 \\
\hline 180 & 1 & 1 & 1 & 1 \\
\hline 220 & 1 & 1 & 1 & 1 \\
\hline 260 & 1 & 1 & 1 & 1 \\
\hline
\end{tabular}

\begin{tabular}{l} 
Detection Limit \\
\hline 0.01 \\
Method for DM analysis \\
\hline Number of simulated data sets \\
\hline 50 \\
\hline Threads \\
\hline $4 \quad$ Go! \\
\hline
\end{tabular}

\section{7}

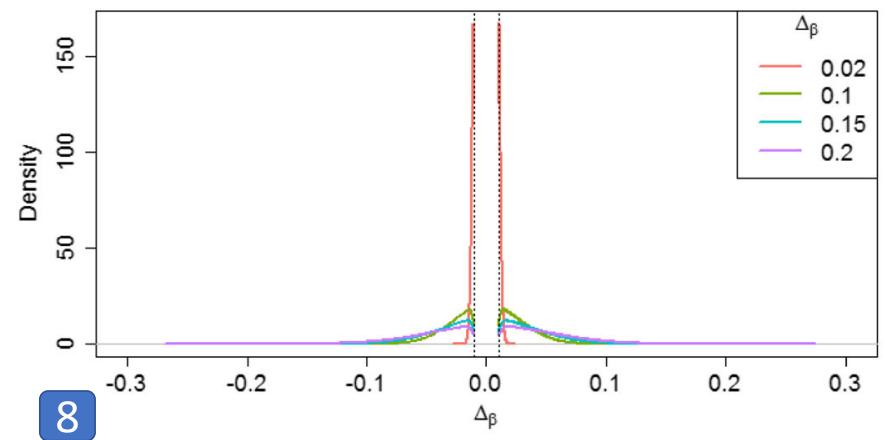

Tissue type $=$ Blood adult

Minimum total sample size $=20$

Maximum total sample size $=260$

Sample gize incremento $=40$

Sanple gize increnento $=40$

Percentage gamplea in group $1=0.5$

Number or Cpas to be tested $=100000$

Target number of $\mathrm{DM} \mathrm{CpGs}=2500$

'Target max Delta' was selected

Target FDR $=0.05$

Detection Limit $=0.01$

Method for DM analysis = 1 imma

Number of simulated data sets $=50$

Threads $=4$

Run time $=49.2$ mins

Fig. 2 (See legend on next page.) 
(See figure on previous page.)

Fig. 2 pwrEWAS Shiny User-Interface. (1) User-specific inputs; (2) Advanced input settings to optimize run time; (3) Link to vignette for detailed description of inputs and outputs, instructions and an example including interpretations of the example results; (4) Power curve as a function of sample size by effect size $\left(\Delta_{\beta}\right) ;(5)$ Estimated power average over simulation by sample size and effect size $\left(\Delta_{\beta}\right) ;(6)$ Probability of detection at least one true positive; (7) Distribution of simulated differences in DNAm $\left(\Delta_{\beta}\right)$ for different target $\Delta_{\beta}{ }^{\prime}{ }^{\prime}$; (8) Log of input parameter and run time

- Tissue type: Blood adult

- Minimum total sample size: 20

- Minimum total sample size: 260

- Sample size increments: 40

- Samples rate for group 1: 0.50

- Number of CpGs tested: 100000

- Target number of DM CpGs: 2500

- Select 'Target max $\Delta$ '

- Target maximal difference in DNAm: 0.02, 0.10, $0.15,0.20$

- Target FDR: 0.05

- Detection Limit: 0.01

- Method for DM analysis: limma

- Number of simulated data sets: 50

- Threads: 4

The results of this power analysis can be found in Fig. 2. To detect differences up to 10,15 and $20 \%$ in CpG-specific methylation across $2500 \mathrm{CpGs}$ between e-cigarette users and non-users with at least $80 \%$ power, we would need about 220, 180 and 140 total subjects, respectively. As expected, $80 \%$ power was not achieved for a difference in DNAm $\leq 2 \%$ for the selected total sample size range. However, it can be observed for this target differences of $2 \%$, that the probability of detecting at least one CpG out of the 2500 differentially methylated CpGs is about $36 \%$ for 20 total patients and virtually $100 \%$ for 60 and more total patients. Because there exists no literature on the magnitude of expected differences in DNAm, a pilot study would be helpful in this hypothetical situation to narrow the range of expected differences to more precisely identify the required sample size to achieve $80 \%$ power.

To evaluate this broad range of sample and effect sizes of this theoretical experiment, pwrEWAS required $\sim 49$ min in total. In general, the computational complexity of pwrEWAS depends on four major components: (1) assumed number and magnitude of sample size(s), (2) number of target $\Delta_{\beta}$ 's (effect sizes), (3) number of CpGs tested, and (4) number of simulated data sets. To enhance the computational efficiency, pwrEWAS allows users to process simulations in parallel. While (1) and (2) are usually dictated by the study to be conducted, (3) and (4) can be modified to either increase the precision of power estimates (increased run time) or reduce the computational burden (decreased precision of estimates). The run time of pwrEWAS for different combinations of sample sizes and effect sizes are provided in Table 3.
As the number of simulated data sets is one of the major components (e.g., item (4), above) affecting the run time of pwrEWAS, it is important to identify a default value that offers a reasonable tradeoff between run time and precision of power estimates. To this end, the variance of power estimates was assessed for a range of simulated data sets (5-100), each repeated 100 times, while keeping the remaining parameters unchanged (Fig. 3a). We ultimately determined the default value for the number of simulated data sets to be 50 , as it appears that simulating additional data sets reduces the variance of power estimates only marginally (Fig. $3 b$ ).

The pwrEWAS package is accompanied by a vignette, which provides a more detailed description of input and output, instructions for the usage, an example, and interpretations of the example results. In addition, a user-friendly R-Shiny point-and-click interface has been developed (Fig. 2) for researchers that are unfamiliar or less comfortable with the $\mathrm{R}$ environment.

\section{Discussion}

In our hypothetical study on the effect of e-cigarette usage on patterns of blood-derived DNAm, we found that $140-220$ total subjects would be needed, depending on the expected effect size. However, these results should be treated with a certain level of caution and considered to be more of a guideline than an exact prescription. Due to computational, memory and storage burden, and simplicity considerations, pwrEWAS involves the random generation methylation $\beta$-values independently across CpGs, which might not hold in real data given previous reports of local correlation in DNAm of nearby CpG sites [35]. Additionally, pwrEWAS assumes CpG-specific homoscedasticity between

Table 3 Run time of pwrEWAS for different combinations of sample sizes and effect sizes

\begin{tabular}{llll}
\hline Total sample sizes & \multicolumn{3}{l}{ Effect sizes $\left(\Delta_{\beta}\right)$} \\
\cline { 2 - 4 } & 0.1 & $0.1,0.2$ & $0.1,0.3,0.5$ \\
\hline 10 & $2 \min 21 \mathrm{~s}$ & $3 \min 11 \mathrm{~s}$ & $3 \min 50 \mathrm{~s}$ \\
100 & $6 \min 22 \mathrm{~s}$ & $7 \min 39 \mathrm{~s}$ & $8 \min 33 \mathrm{~s}$ \\
500 & $24 \min 43 \mathrm{~s}$ & $27 \min 36 \mathrm{~s}$ & $29 \min 22 \mathrm{~s}$ \\
$10-100$ (increments of 10) & $9 \min 40 \mathrm{~s}$ & $16 \min 34 \mathrm{~s}$ & $23 \min 44 \mathrm{~s}$ \\
$300-500$ (increments of 100) & 27 min 58s & 30 min 01 s & 52 min 00s
\end{tabular}

In all scenarios presented the number of tested $\mathrm{CpGs}$ was assumed to be 100,000 , number of simulated data sets was 50 , and the method to perform the differential methylation analysis as limma. A total of 6 clusters/threads were used 


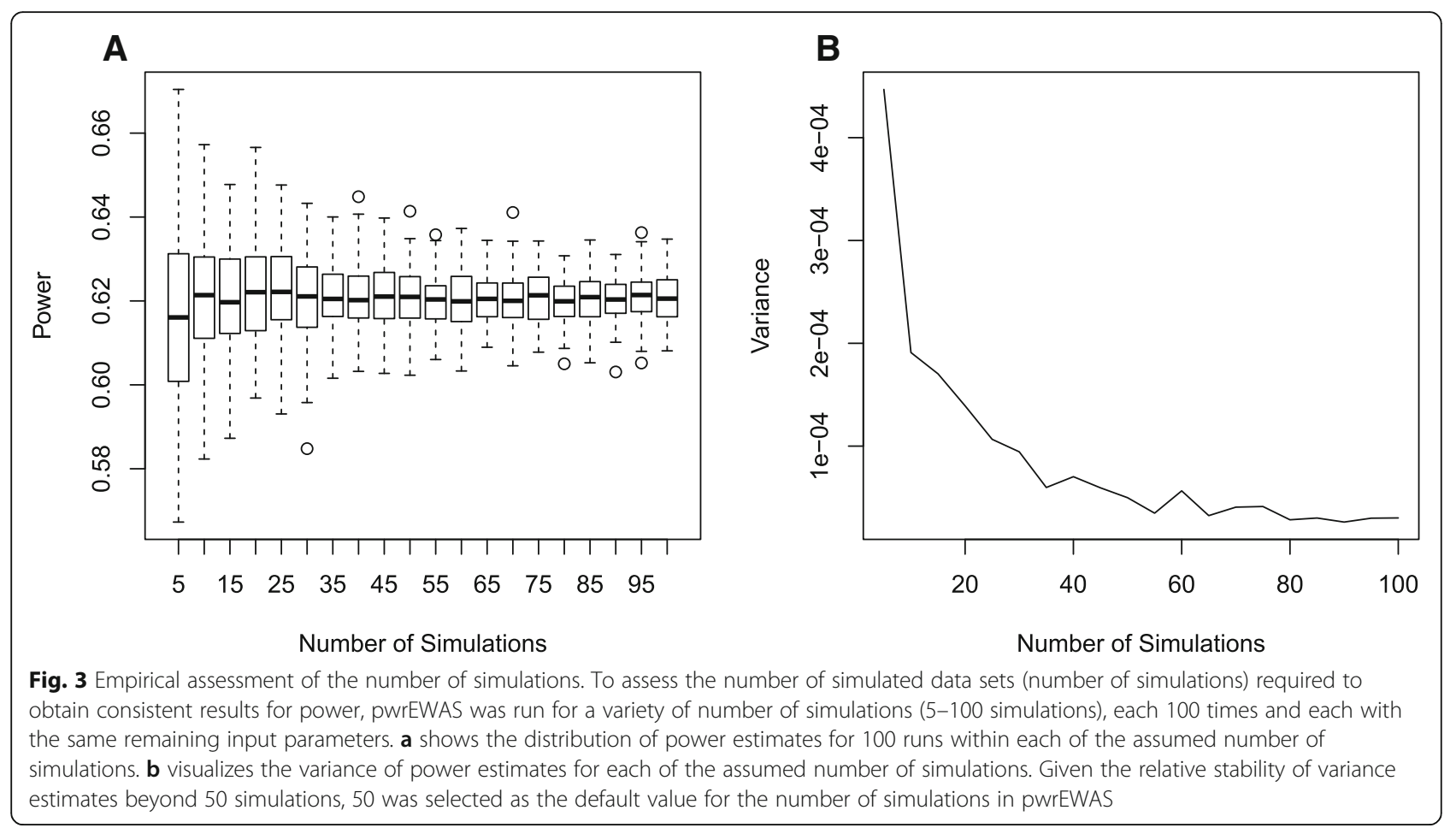

both comparator groups, that is CpG-specific variances are assumed to be identical between both groups. However, CpG-specific variances have been reported to change depending on exposure(s) and phenotype(s) [36, 37]. Violations of CpG-specific homoscedasticity can result in inflated estimates of statistical power and produce overly optimistic sample sizes, however identifying the magnitude of changes in variances depending on exposure(s) and phenotype(s) in advance of the study can be very challenging. Further, the expected difference in DNAm between both groups $\left(\Delta_{\beta}\right)$ is assumed to come from a truncated normal. This assumption seems to hold, at least approximately, based on observed distributions of differences in DNAm across a variety of studies. Additional limitations of pwrEWAS include: two group comparison, selection of methods for differential methylation analysis, and selection of tissue types specific reference data.

Despite the above limitations, pwrEWAS is to our knowledge, the first publicly available tool to formally address the issue of power evaluation in the context of EWAS. Further opportunities for the extension of pwrEWAS include the implementation of additional methods for differential methylation analysis (e.g., linear regression for continuous phenotype(s)/exposure(s), Cox-proportional hazards models or relevant models for handling time-to-event outcomes, etc.), allowing multiple group comparisons, providing the opportunity for researcher to upload different reference data (tissue type(s) specific to their study), and addressing the potential change of CpG dispersion due to phenotype(s) and/or exposure(s).

\section{Conclusion}

When designing an EWAS, consideration of statistical power should play a central role in selecting the appropriate sample size to address the question(s) of interest. Under- and overpowered studies waste resources and even risk failure of the study. With pwrEWAS we present a user-friendly power evaluation tool with the goal of helping researchers in the design and planning of their EWAS.

\section{Availability and requirements \\ Project name: pwrEWAS. \\ Project homepage: https://github.com/stefangraw/ pwrEWAS}

Operating systems: Platform independent.

Programming language: $R$.

License: Artistic-2.0.

Any restrictions to use by non-academics: none.

\section{Additional files}

Additional file 1: Derivation for upper and lower bound of $\Delta_{,} \mathrm{CpG}$ specific differences in mean methylation between two compared groups. (DOCX $28 \mathrm{~kb}$ )

Additional file 2: Supplementary Figure 1 and Supplementary Figure 2. (DOCX $639 \mathrm{~kb}$ )

\section{Abbreviations}

DNAm: DNA methylation; EWAS: Epigenome-Wide Association Study; FDC: False Discovery Cost; FDR: False Discovery Rate 


\section{Acknowledgements}

We would like to extend our gratitude to Dr. Dong Pei, Lisa Neums, Richard Meier, Qing Xia, Shachi Patel, Duncan Rotich and Dinesh Pal Mudaranthakam of the Department of Department of Biostatistics \& Data Science at the University of Kansas Medical Center for their constructive feedback on pwrEWAS.

\section{Funding}

Research reported in this publication was supported by the Kansas IDeA Network of Biomedical Research Excellence Bioinformatics Core, supported in part by the National Institute of General Medical Science award P20GM103428. Funding from the aforementioned grant was used to finance access to high-performance computing, which was used in the development and testing of pwrEWAS.

\section{Availability of data and materials}

$\mathrm{R}$ implementation of pwrEWAS and a vignette are available at https://github. com/stefangraw/pwrEWAS.

\section{Authors' contributions}

SG developed the methodology, implemented the pwrEWAS package and wrote the manuscript. RH managed the acquisition and processing of the reference data sets and edited the manuscript. JT edited the manuscript. DK supervised the implementation and edited the manuscript. All authors read and approved the final version of the manuscript.

\section{Ethics approval and consent to participate}

Not applicable.

\section{Consent for publication}

Not applicable.

\section{Competing interests}

The authors declare that they have no competing interests.

\section{Publisher's Note}

Springer Nature remains neutral with regard to jurisdictional claims in published maps and institutional affiliations.

\section{Author details}

'Department of Biostatistics \& Data Science, University of Kansas Medical Center, Kansas City, KS, USA. ${ }^{2}$ Department of Cancer Biology, University of Kansas Medical Center, Kansas City, KS, USA.

\section{Received: 18 October 2018 Accepted: 10 April 2019}

Published online: 29 April 2019

\section{References}

1. Bibikova M, Le J, Barnes B, Saedinia-Melnyk S, Zhou LX, Shen R, Gunderson $\mathrm{KL}$. Genome-wide DNA methylation profiling using Infinium (R) assay. Epigenomics. 2009;1(1):177-200.

2. Pidsley R, Zotenko E, Peters TJ, Lawrence MG, Risbridger GP, Molloy P, Van Djik S, Muhlhausler B, Stirzaker C, Clark SJ. Critical evaluation of the Illumina MethylationEPIC BeadChip microarray for whole-genome DNA methylation profiling. Genome Biol. 2016;17.

3. Kulis M, Esteller M. DNA methylation and Cancer. Adv Genet. 2010;70:27-56

4. Robertson KD. DNA methylation and human disease. Nat Rev Genet. 2005; 6(8):597-610.

5. Martin EM, Fry RC. Environmental influences on the epigenome: exposureassociated DNA methylation in human populations. Annu Rev Public Health. 2018;39:309-33.

6. Yang XJ, Lay F, Han H, Jones PA. Targeting DNA methylation for epigenetic therapy. Trends Pharmacol Sci. 2010;31(11):536-46.

7. Saadati M, Benner A. Statistical challenges of high-dimensional methylation data. Stat Med. 2014;33(30):5347-57

8. Teschendorff AE, Relton CL. Statistical and integrative system-level analysis of DNA methylation data. Nat Rev Genet. 2018;19(3):129-47.

9. Guo Y, Zhao S, Li Cl, Sheng Q, Shyr Y. RNAseqPS: a web tool for estimating sample size and power for RNAseq experiment. Cancer Inform. 2014; 13(Suppl 6):1-5.
10. Ching T, Huang SJ, Garmire LX. Power analysis and sample size estimation for RNA-Seq differential expression. Rna. 2014;20(11):1684-96.

11. Wu H, Wang C, Wu ZJ. PROPER: comprehensive power evaluation for differential expression using RNA-seq. Bioinformatics. 2015;31(2):233-41.

12. Skol AD, Scott $L$, Abecasis GR, Boehnke M. Joint analysis is more efficient than replication-based analysis for two-stage genome-wide association studies. Nat Genet. 2006;38(2):209-13.

13. Blaise BJ, Correia G, Tin A, Young JH, Vergnaud AC, Lewis M, Pearce JT, Elliott $P$, Nicholson JK, Holmes $E$, et al. Power analysis and sample size determination in metabolic phenotyping. Anal Chem. 2016;88(10):5179-88.

14. Feng S, Wang SC, Chen CC, Lan L. GWAPower: a statistical power calculation software for genome-wide association studies with quantitative traits. BMC Genet. 2011;12.

15. Syed $H$, Jorgensen AL, Morris AP. SurvivalGWAS_power: a user friendly tool for power calculations in pharmacogenetic studies with "time to event" outcomes. Bmc Bioinformatics. 2016;17.

16. Li DM, Xie ZD, Le Pape M, Dye T. An evaluation of statistical methods for DNA methylation microarray data analysis. Bmc Bioinformatics. 2015;16.

17. Siegmund KD. Statistical approaches for the analysis of DNA methylation microarray data. Hum Genet. 2011;129(6):585-95.

18. Michels KB, Binder AM, Dedeurwaerder S, Epstein CB, Greally JM, Gut I, Houseman EA, Izzi B, Kelsey KT, Meissner A, et al. Recommendations for the design and analysis of epigenome-wide association studies. Nat Methods. 2013;10(10):949-55.

19. Tsai PC, Bell JT. Power and sample size estimation for epigenome-wide association scans to detect differential DNA methylation. Int J Epidemiol. 2015;44(4):1429-41.

20. Rakyan VK, Down TA, Balding DJ, Beck S. Epigenome-wide association studies for common human diseases. Nat Rev Genet. 2011;12(8):529-41.

21. Wang S. Method to detect differentially methylated loci with case-control designs using Illumina arrays. Genet Epidemiol. 2011;35(7):686-94.

22. Lokk K, Modhukur V, Rajashekar B, Martens K, Magi R, Kolde R, Koltsina M, Nilsson TK, Vilo J, Salumets A, et al. DNA methylome profiling of human tissues identifies global and tissue-specific methylation patterns. Genome Biol. 2014;15(4).

23. Langie SAS, Moisse M, Declerck K, Koppen G, Godderis L, Vanden Berghe W, Drury S, De Boever P. Salivary DNA methylation profiling: aspects to consider for biomarker identification. Basic Clin Pharmacol. 2017;121:93-101.

24. Du P, Zhang XA, Huang CC, Jafari N, Kibbe WA, Hou LF, Lin SM. Comparison of Beta-value and M-value methods for quantifying methylation levels by microarray analysis. Bmc Bioinformatics. 2010;11.

25. Logue MW, Smith AK, Wolf EJ, Maniates H, Stone A, Schichman SA, McGlinchey RE, Milberg W, Miller MW. The correlation of methylation levels measured using Illumina 450K and EPIC BeadChips in blood samples. Epigenomics. 2017;9(11):1363-71

26. Ritchie ME, Phipson B, Wu D, Hu YF, Law CW, Shi W, Smyth GK. Limma powers differential expression analyses for RNA-sequencing and microarray studies. Nucleic Acids Res. 2015;43(7).

27. Barfield RT, Kilaru V, Smith AK, Conneely KN. CpGassoc: an R function for analysis of DNA methylation microarray data. Bioinformatics. 2012;28(9):1280-1.

28. Wilhelm-Benartzi CS, Koestler DC, Karagas MR, Flanagan JM, Christensen BC, Kelsey KT, Marsit CJ, Houseman EA, Brown R. Review of processing and analysis methods for DNA methylation array data. Brit J Cancer. 2013;109(6): 1394-402.

29. Benjamini $Y$, Hochberg Y. Controlling the false discovery rate - a practical and powerful approach to multiple testing. J Roy Stat Soc B Met. 1995;57(1): 289-300.

30. Chen H, Li G, Chan YL, Chapman DG, Sukjamnong S, Nguyen T, Annissa T, McGrath KC, Sharma P, Oliver BG. Maternal E-cigarette exposure in mice alters DNA methylation and lung cytokine expression in offspring. Am J Resp Cell Mol. 2018:58(3):366-77.

31. Nguyen T, Li GE, Chen H, Cranfield CG, McGrath KC, Gorrie CA. Maternal Ecigarette exposure results in cognitive and epigenetic alterations in offspring in a mouse model. Chem Res Toxicol. 2018;31(7):601-11.

32. Ambatipudi S, Cuenin C, Hernandez-Vargas H, Ghantous A, Le Calvez-Kelm F, Kaaks R, Barrdahl M, Boeing H, Aleksandrova K, Trichopoulou A, et al. Tobacco smoking-associated genome-wide DNA methylation changes in the EPIC study. Epigenomics. 2016:8(5):599-618.

33. Joehanes R, Just AC, Marioni RE, Pilling LC, Reynolds LM, Mandaviya PR, Guan W, Xu T, Elks CE, Aslibekyan S, et al. Epigenetic signatures of cigarette smoking. Circ Cardiovasc Genet. 2016;9(5):436-47. 
34. Zeilinger S, Kuhnel B, Klopp N, Baurecht H, Kleinschmidt A, Gieger C, Weidinger S, Lattka E, Adamski J, Peters A, et al. Tobacco smoking leads to extensive genome-wide changes in DNA methylation. PLoS One. 2013;8(5).

35. Zhang WW, Spector TD, Deloukas P, Bell JT, Engelhardt BE. Predicting genome-wide DNA methylation using methylation marks, genomic position, and DNA regulatory elements. Genome Biol. 2015;16.

36. Hansen KD, Timp W, Bravo HC, Sabunciyan S, Langmead B, McDonald OG, Wen B, Wu H, Liu Y, Diep D, et al. Increased methylation variation in epigenetic domains across cancer types. Nat Genet. 2011;43(8):768-75.

37. Teschendorff AE, Jones A, Fiegl H, Sargent A, Zhuang JJ, Kitchener HC, Widschwendter M. Epigenetic variability in cells of normal cytology is associated with the risk of future morphological transformation. Genome Med. 2012;4.

38. Hong SR, Jung SE, Lee EH, Shin KJ, Yang WI, Lee HY. DNA methylationbased age prediction from saliva: high age predictability by combination of 7 CpG markers. Forensic Sci Int Genet. 2017;29:118-25.

39. Matsunaga A, Hishima T, Tanaka N, Yamasaki M, Yoshida L, Mochizuki M Tanuma J, Oka S, Ishizaka Y, Shimura M, et al. DNA methylation profiling can classify HIV-associated lymphomas. Aids. 2014;28(4):503-10.

40. Kawai T, Yamada T, Abe K, Okamura K, Kamura H, Akaishi R, Minakami H, Nakabayashi K, Hata K. Increased epigenetic alterations at the promoters of transcriptional regulators following inadequate maternal gestational weight gain. Sci Rep-Uk. 2015;5.

41. Horvath S, Erhart W, Brosch M, Ammerpohl O, von Schonfels W, Ahrens M, Heits N, Bell JT, Tsai PC, Spector TD, et al. Obesity accelerates epigenetic aging of human liver. Proc Natl Acad Sci U S A. 2014;111(43):15538-43.

42. Mclnnes T, Zou DH, Rao DS, Munro FM, Phillips VL, McCall JL, Black MA, Reeve AE, Guilford PJ. Genome-wide methylation analysis identifies a core set of hypermethylated genes in CIMP-H colorectal cancer. BMC Cancer. 2017;17.

43. Kular L, Liu Y, Ruhrmann S, Zheleznyakova G, Marabita F, Gomez-Cabrero D, James T, Ewing E, Linden M, Gornikiewicz B, et al. DNA methylation as a mediator of HLA-DRB1(star) 15:01 and a protective variant in multiple sclerosis. Nat Commun. 2018:9.

44. Liu Y, Aryee MJ, Padyukov L, Fallin MD, Hesselberg E, Runarsson A, Reinius L, Acevedo N, Taub M, Ronninger M, et al. Epigenome-wide association data implicate DNA methylation as an intermediary of genetic risk in rheumatoid arthritis. Nat Biotechnol. 2013:31(2):142-7.

45. Urdinguio RG, Torro MI, Bayon GF, Alvarez-Pitti J, Fernandez AF, Redon P, Fraga MF, Lurbe E. Longitudinal study of DNA methylation during the first 5 years of life. J Transl Med. 2016;14.

46. Markunas CA, Wilcox AJ, Xu ZL, Joubert BR, Harlid S, Panduri V, Haberg SE, Nystad W, London SJ, Sandler DP, et al. Maternal age at delivery is associated with an epigenetic signature in both newborns and adults. PLoS One. 2016;11(7).

47. Langie SAS, Moisse M, Szic KSV, Van der Plas E, Koppen G, De Prins S, Louwies T, Nelen V, Van Camp G, Lambrechts D, et al. GLI2 promoter hypermethylation in saliva of children with a respiratory allergy. Clin Epigenetics. 2018;10.

48. Zhang YH, Petropoulos S, Liu JH, Cheishvili D, Zhou R, Dymov S, Li K, Li N, Szyf M. The signature of liver cancer in immune cells DNA methylation. Clin Epigenetics. 2018;10.

Ready to submit your research? Choose BMC and benefit from:

- fast, convenient online submission

- thorough peer review by experienced researchers in your field

- rapid publication on acceptance

- support for research data, including large and complex data types

- gold Open Access which fosters wider collaboration and increased citations

- maximum visibility for your research: over $100 \mathrm{M}$ website views per year

At BMC, research is always in progress.

Learn more biomedcentral.com/submissions 